\title{
Using Haptic Feedback in Human Robotic Swarms Interaction
}

\author{
Steven Nunnally, Phillip Walker, Mike Lewis \\ University of Pittsburgh
}

\author{
Nilanjan Chakraborty, Katia Sycara \\ Carnegie Mellon University
}

\begin{abstract}
Robotic swarms display emergent behaviors that are robust to failure of individual robots, although they can not necessarily accomplish complex tasks with these behaviors. The research objective is to make use of their robust behaviors to accomplish complex tasks in many types of environments. For now, it is difficult to affect swarm "goals", and therefore difficult them to direct to perform complex tasks. The extant literature on Human Swarm Interaction (HSI) focuses on demonstrating the usefulness of human operator inputs for swarms to accomplish complex tasks. The human typically gets visual feedback of the state of the swarm and influences the robots through a computer interface. This paper presents a user study investigating the effectiveness of haptic feedback in improving HSI. We use methods developed in studies using haptics in multi-robot systems (where the communication and structure is very rigid) and potential field algorithms developed for fully-autonomous swarms to determine the benefits of haptic feedback from the semi-autonomous control algorithm. In some environments, haptic feedback proved beneficial whereas in other environments haptic feedback did not improve performance over visual feedback alone. However, presence of haptic feedback did not degrade the performance under any of the experimental conditions. This supports our working hypothesis that haptic feedback is potentially useful in HSI.
\end{abstract}

\section{INTRODUCTION}

Swarm robotics is characterized by using simple robots in large numbers to accomplish complex tasks. The emergent behaviors, based on the local interactions between the robots and their environment, allow the swarm to accomplish tasks even with the sensor and computation limitations necessary to produce a large group of robots affordably. Unfortunately, the algorithms producing the emergent behaviors are often only guaranteed to succeed in very controlled environments, which is not practical in real world applications. Real world applications include: search and rescue operations, military surveillance, or first responder assistance (Kira, 2009; Bashyal, 2008; Naghsh, 2008; Kolling, 2012). For instance, a swarm of robots could cover a disaster zone to search for radiation sources. This gives the decision makers more information to determine the risk of sending first humans into the area. The environments in these applications are often cluttered and unpredictable, so the swarm algorithms may need to be supplemented with effective human interaction to guide the swarm through these difficult scenarios.

The extant literature on Human Swarm Interaction (HSI) focuses on finding methods for the user to influence the swarm to effectively achieve a goal using an interface that gives perfect and complete information about the swarm and the environment from a bird's eye view (Nunnally, 2012; Walker, 2012; Kira, 2009; Bashyal, 2008; Goodrich, 2011; Kolling, 2012). The contributions are new swarm algorithms and ways in which a human operator can add utility to this new algorithm. Some HSI studies demonstrate the possibilities of decentralized swarms with few robots (Kira, 2009). Others have built models of the operator, influencing a decentralized swarm (Cummings, 2004; Kira, 2009; Coppin, 2012). Some studies look at specific applications and analyze techniques for accomplishing tasks through simulated environments (Nunnally, 2012; Walker, 2012; Bashyal, 2008; Naghsh, 2008; Kolling, 2012; Coppin, 2012).
In the HSI literature, the swarm state is usually fed back through the visual channel. Some formation control experiments (Secchi, 2012; Franchi, 2011; Rodriguez-Seda, 2010; Lee, 2011) in which robots maintain rigid spatial relations, have explored the use of haptic feedback devices to give users tactile information about the state of the robots and also allowed the users to control the system by giving inputs through the haptic device. The force fed back to the humans contained information about the deviation of the centroid of the formation from a planned path and proximity of robots to obstacles. This research has focused on designing force feedback laws to make the overall system robust to communication delays and was tested on systems with few robots in simulation (Secchi, 2012; Lee, 2011). Haptic feedback laws for dealing with changing topologies as the robot system moves through obstacle filled environments, so that the robots may traverse the obstacles quickly without collisions, have also been developed (Franchi, 2011; Rodriguez-Seda, 2010).

Although the use of haptic control has demonstrated success for formation control in completing tasks like information foraging where there are rigid structural constraints on the robots, it is not clear whether haptic feedback information would be useful in controlling swarms. Therefore, in this paper, our goal is to study the utility of haptic feedback in influencing decentralized swarm robotic systems, where the tasks are specified at a high level (e.g., find as many targets as you can in a given environment) and the connection topology of the swarms is flexible. The task for operators is to find targets in four obstacle-filled environments with different features. We use a between-subjects design for this study, i.e. half of the subjects are given haptic feedback to control the swarm along with visual feedback about the positions of the robots, and half of them are only given visual feedback about the position of the robots. The experiment, interface, and robot control algorithms are explained in the EXPERIMENT section. The RESULTS section discusses the 


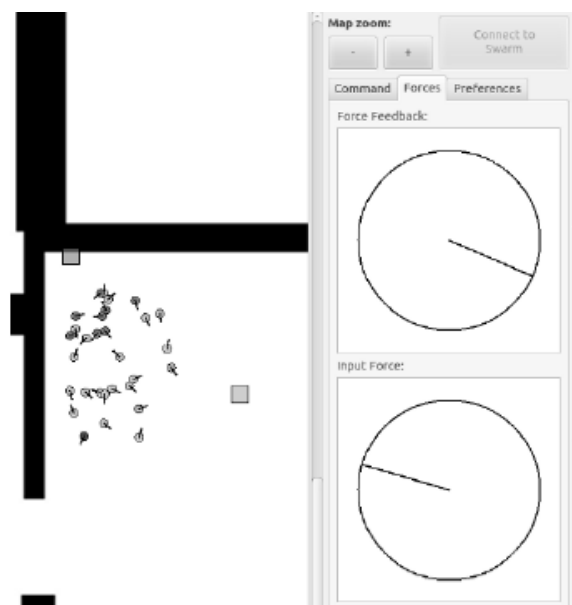

Figure 1 The GUI used for every condition of the study. The left side shows the robots' estimated positions, obstacles, and marked targets. The right side shows the Force Feedback and the Input Force.

results of the user study. Finally, the findings and future works of the study are presented in the DISCUSSION section.

\section{EXPERIMENT}

\section{Interface}

Stage v. 3.2.2 (Gerkey 2003) was used to simulate the environment, the targets, and a swarm of 30 P2AT robots, which is a simulated, four-wheeled, skid-steered robot. The graphical user interface and robot controllers are implemented using the Robot Operating System (ROS) (Quigley 2009). The interface for this study is similar to that of (Nunnally, 2012; Walker, 2012), see Figure 1. The users have a bird's eye view of the simulated area. The participant can translate and zoom the view port using the mouse. Robots appear as circles with lines pointing in the direction of their heading. Each robot turns the color of a target if it detects one within its sensing range. Targets are only marked on the interface and counted as found when a five of robots detect the target. This compensates for sensor error, as explained in the next section. The threshold requires the users to maintain a cohesive swarm in order to complete the task. Obstacles, if shown, appear in the interface in black, while traversable areas are white.

A Phantom Omni device is used for participant input by translating the device's coordinate system to a frame on the desk next to the monitor, see Figure 2. The vector from the center of the circle on the desk to the end effector of the Phantom device gives the human input force described in the next section. The Input Force panel to the right of the view port displays the vector of the input force taken from the Phantom device so the participant does not have to look away from the screen to adjust their input. The vector is broadcast to the swarm and used to influence their path, as shown with $F_{h}$ in the next subsection. The participants are made aware of the difficulty of controlling multiple swarm groups with only one input source without a method of selection during their training period so that they are encouraged to keep the communication graph of the swarm connected. The Force Feedback panel above the Input Force panel shows feedback

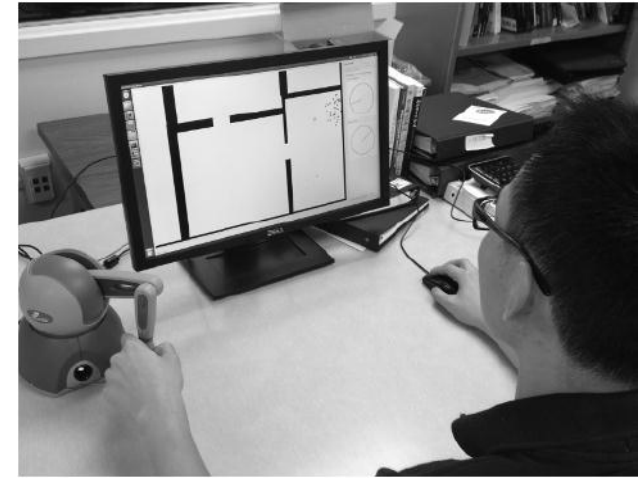

Figure 2 Participants used an Omni Phantom device and mouse to influence the swarm and manipulate the interface respectively.

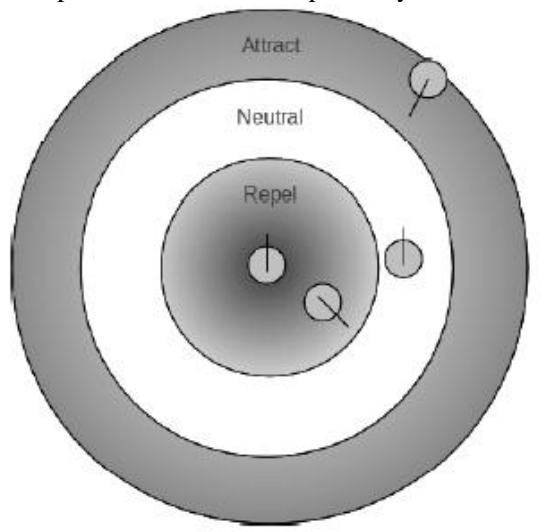

Figure 3 The closer the robots are, the stronger the repulsive force, the further the robots are, the strong the attractive force. The robots stabilize to the neutral zone without $\boldsymbol{F}_{o}$ and $\boldsymbol{F}_{h}$.

from obstacles which will be discussed as $F_{O}$ in the next subsection.

\section{Robot Control Algorithm}

The robot control algorithm is based on the vector field algorithm used in (Howard, 2002) which uses repulsive forces from other robots and obstacles to deploy robots for maximum covering. An attractive force is introduced between robots to help maintain connectivity. The resulting algorithm is similar to Craig's (1986) "boids" algorithm for simulating flocking. Each robot determines its motion by calculating the potential field given by $\boldsymbol{F}=\boldsymbol{F}_{o}+\boldsymbol{F}_{r}+\boldsymbol{F}_{h}$ with the forces due to obstacles, other robots, and human influence, respectively. The robot moves in the direction of this vector and the speed given by the magnitude, although the magnitude is generally greater than the robot's top speed. More precisely, let $o \in V\left(q_{i}\right)$ be all obstacles in range of robot at location $q_{i}$ and $r_{o}=\left|q_{i}-q(o)\right|$ and $\boldsymbol{r}_{o}=q_{i}-q(o)$, then:

$$
\boldsymbol{F}_{o}=-k_{o} \sum_{o \in V\left(q_{i}\right)} \frac{1}{r_{o}^{2}} \frac{r_{o}}{r_{o}}
$$

Similarly, let $r \in V\left(q_{i}\right)$ be all robots in range $r_{i}$ of $q_{i}$ and $r_{r}=\left|q_{i}-q(r)\right|$ and $\boldsymbol{r}_{r}=q_{i}-q(r)$ and $n_{b}$ be the beginning radius of the neutral zone and $n_{e}$ be the ending radius of the neutral zone, then: 


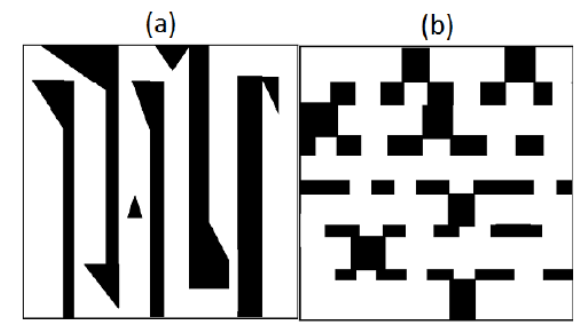

(c)

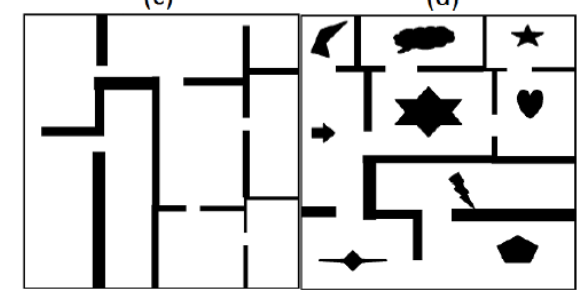

Figure 4 Four environments used in the study. The robots always started in the lower right corner: (a) Math, (b) Speed, (c) Control, and (d) Hidden (note that the participants cannot see these obstacles).

$$
\boldsymbol{F}_{r}= \begin{cases}-k_{r} \sum_{r \in V\left(q_{i}\right) \frac{1}{r_{r}^{2}} \frac{r_{r}}{r_{r}},} & q_{i}<n_{b} \\ k_{r} \sum_{r \in V\left(q_{i}\right)} \frac{1}{\left(r_{i}-r_{r}\right)^{2}} \frac{r_{r}}{\left(r_{i}-r_{r}\right)}, & q_{i}>n_{e}\end{cases}
$$

This disperses the robots, while the attraction dissuades breaking the communication limitation of 4 meters, see Figure 3 . Finally, let $h$ be the input force vector from the Phantom device and $r_{h}=|h|$ and $\boldsymbol{r}_{h}=h$ and $h_{\max }$ be the magnitude of the maximum allowed force, then:

$$
\boldsymbol{F}_{h}=k_{h} \frac{1}{\left(h_{\max }-r_{h}\right)^{2}} \frac{r_{h}}{\left(h_{\max }-r_{h}\right)}
$$

The constant values in this study are $k_{o}=5, k_{r}=3$, $k_{h}=7, n_{b}=2.5, n_{e}=3.0, h_{\max }=4$. The $\max$ speed is 0.6 $\mathrm{m} / \mathrm{s}$. This is the normal for most environments, although these values change for the Speed environment and will be discussed in the next subsection. This balances good area coverage with the risk of breaking communication before attraction can take effect beyond the neutral zone values. The robot senses other robots and obstacles within a 360 degree field of view using a 4 degree resolution. Average $\boldsymbol{F}_{o}$ across all robots is shown in the Force Feedback panel in Figure 1 for all conditions and felt in the Phantom device, if the participant is in the haptics condition.

The robots' faulty target sensors miss at a rate of $p_{n}=$ $\left(1-\frac{r-d}{r}\right)^{2}$ where $d$ is the distance to the object, $r$ is the range of the sensor, and $\alpha$ is the decay rate set to 4 . The sensor may also report a false positive. Occurrences of false positives were recalculated at an interval $t_{r}$ equal to some randomly sampled value between 6 and 10 seconds for each of the 30 robots. When a false positive occurred, an imaginary target was reported at a randomly chosen position at the edge of the target sensor's range. The automated target marking system only marks a target when five robots simultaneously sense a target, using redundancy to compensate for errors from faulty sensors. This error and method of overcoming the error is used as to encourage participants to keep the swarm connected through its communication graph.

\section{Environments}

The participants worked to find as many targets as possible in four different obstacle filled environments. The starting position of the robots was in the bottom right corner, and each environment contained 60 targets.

The first environment is a corridor maze called Math, see Figure 4(a). The width of the halls was such that the robots could mark targets along the walls if the swarm traveled down the center, but there were choke points and traps which could slow the participant down. The participant was instructed that the optimal strategy is to avoid the traps. Single digit addition problems blocked the view port every ten to fifteen seconds, leaving only the side panel with the Force Feedback and Input vectors visible, and remained up until participants responded by keyboard correctly. Normal operation occurred behind the math problem. This condition corresponds to a situation in which navigation is a secondary task and an infrequent primary task may require full visual attention at certain times, i.e. checking video surveillance while directing the swarm.

The second environment is structured around many decisions called Speed, see Figure 4(b). The dead ends and intersections require focus and decision making for operators to determine the best path to explore as much area as possible in order to find as many of the targets as possible. The distinctive feature of this environment is greater speed which required changes in the force constants to avoid wall collisions. The speed was set to $1.0 \mathrm{~m} / \mathrm{s}$ and the constants were set to $k_{o}=10, k_{r}=4, k_{h}=5$. The change in speed is to further the attention and focus required to make quick decisions when covering the environment. This new configuration created a volatile swarm which was more likely to break up around obstacles when pushing against them.

The third environment is a structured office type environment without obstacles in the rooms called Control, see Figure 4(c). This was used as the control world without special features. Participants were told to explore the rooms thoroughly before moving to the next one, as doorways slow the swarm down and spread them out.

The final environment is structured with obstacles with edges and concave corners requiring exploration called Hidden, see Figure 4(d). In this environment the obstacles are hidden, so the participants had no idea where the obstacles were, except by observed behavior, side panel information, and haptic feedback for the haptic condition. The participants were told it was an office structure with obstacles in the room, and that the best strategy was to sweep the rooms, avoiding a lot of force from the walls and then finding an exit. They were also instructed to use marked targets as landmarks near exits in case the room was a dead end.

\section{Participants}

The study was a between-subjects design. Thirty-two paid student participants from the University of Pittsburgh were 


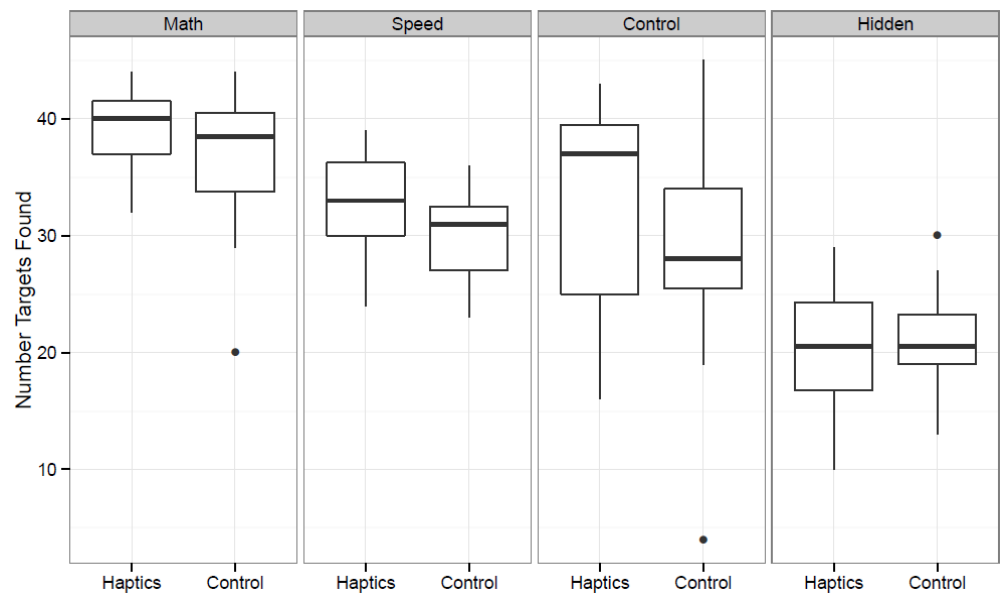

Figure 5 Box plot around the median number of targets found in each trial across conditions and environments. Each box represents sixteen trials.

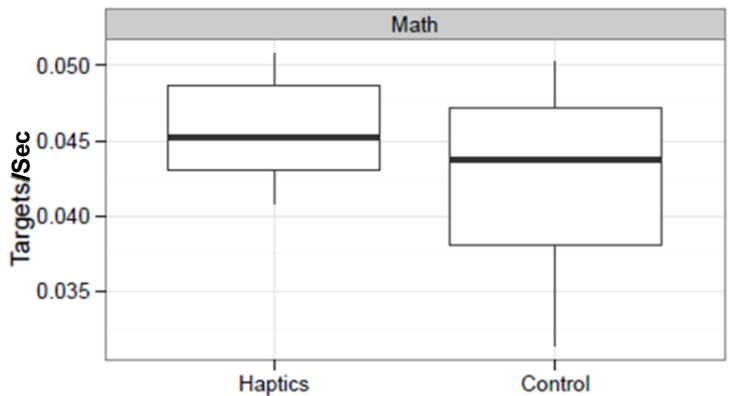

Figure 6 Box plot around the median performance efficiency in the Math environment. Each box represents sixteen trials.

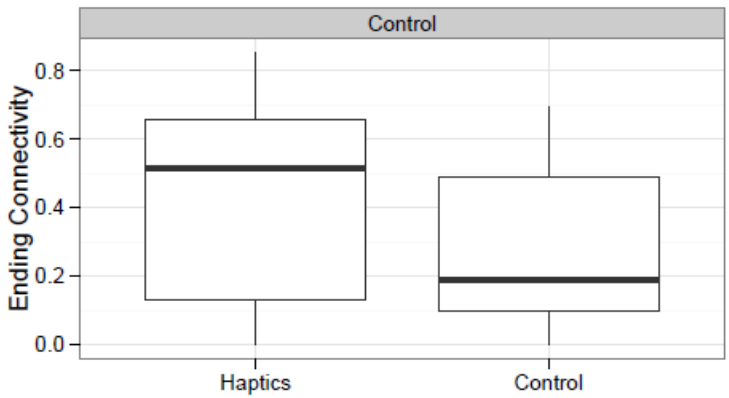

Figure 7 Box plot around the median of the ending measure of connectivity in the Control environment. Each box represents sixteen trials.

divided into two groups. One group received visual and physical force feedback in the Phantom device as described above, hereafter referred to as the haptics group. The control group received only visual feedback. All other variables remained constant. Participants were given an explanation of the robot control algorithms, interface, and importance of a cohesive swarm, after which they were given a 10 minute practice period to gain experience and ask questions. As preparation for the Math environment, a problem popped up every thirty to sixty seconds. Environments were presented in random order, and the participants were given fifteen minutes in each environment to find as many targets as possible.

\section{RESULTS}

Results are presented for performance and measures of connectivity in swarm communication topology. Comparisons between conditions and environments were tested with an
ANOVA. Because of excessive variability in our data and lack of prior work in the area we have chosen to report marginal differences that may suggest possible directions for future research as well as substantiated differences meeting conventional criteria.

In Figure 5, the Control and Speed environments both show a marginally significant performance increase for the haptics condition, $(\mathrm{F}=2.653$ and $\mathrm{F}=2.606$, respectively $\mathrm{p}<0.12$ ). By contrast, no differences were attributable to the use of haptics in the Math or Hidden environments. Because participants in the Haptics condition were observed to explore more rapidly but to sometimes get bogged down retracing their path, we introduced targets/time as a measure of efficiency. This efficiency measure divides the number of targets found by the time at which the last target was reported thus removing the influence of swarms which become stuck or lose time redundantly covering an already explored area at the end of the session. Using this measure, the Math environment did show an advantage for a marginally significant performance efficiency increase with the haptics condition, $(\mathrm{F}=3.547, \mathrm{p}<0.07)$, see Figure 6 .

These results show that in office and simple intersection environments, even with a volatile swarm, participants may have performed slightly better with haptic feedback than without. As for the Math environment, it was observed that most participants were able to make it to the bottom of the fourth corridor and for most, the time ended when traversing the two choke points to enter into the fifth hallway. The control condition must have caught up at this point, which was too difficult to traverse well. This could explain the similar performances between the conditions with the number of targets found and would show a performance increase in the target finding rate. Therefore, the haptics condition also performed better up until the difficult obstacle. Further investigation is required to understand the significance of this finding. Counter-intuitively, the Hidden environment did not show any significant difference between the conditions. The most probable reason is misunderstanding of the prevailing strategy, unfamiliarity of the controls, or that the environment may not be well suited for haptic feedback in this scenario. Further investigation should see why tactile information does not aide the participant in that environment. 
We use a variation of Fiedler's Number to investigate the swarm's communication graph connectivity, since the task was set up in a way to create benefits to those who kept the swarm more connected. To overcome the limitation that a disconnected graph is zero for Fiedler's Number, the number was calculated for the largest connected component, based on the robots' communication range, as long as it was greater or equal to half of the group. Figure 7 shows a marginal increase in the ending connectivity in the haptics condition for the Control environment, $(F=2.757, p<0.11)$. No difference was found for the other environments, probably because so many trials ended with a connectivity of zero.

This study shows the haptic condition aiding connectivity. The doorways were the only obstacles in the Control environment that might separate or disconnect parts of the swarm. The increased performance shows that the haptics condition must have traversed many doors to cover enough area to find more targets. The most likely reason for the increased connectivity for the haptics condition is that the feedback must have helped the operators move through the center of the doorway, creating the smallest $F_{o}$ possible to keep the swarm better connected. This traversal would also be more efficient explaining the performance increase.

\section{DISCUSSION}

The study showed some utility in using haptic feedback with HSI in all scenarios except the hidden environment. Even then, the presence of haptic feedback never impeded the performance. Haptic feedback helped in simple office environments and even in maze-like environments where the volatility of the swarm is increased, and thus the swarm is more difficult to keep together. A distracting primary task showed an increase in performance efficiency as well; showing the many different ways haptic feedback might help a newly trained human operator to influence a decentralized group of robots to complete a task.

The results indicate that haptic feedback increased connectivity in environments with nothing but rooms and doors. It did not increase connectivity in other cases, however, so there are strategies or environmental traits that affect this measure that should be extracted from the data, or a new study should be used to understand the effect of haptic feedback on swarm topology. The discovery could help the operator influence the swarm to better achieve the goal, whether that is by breaking up the swarm to explore more area or by increasing connectivity to gain better resolution of an area.

Further investigation is needed to determine the lack of effects in the Hidden environment. More training could help the operator's use of the haptic feedback when obstacles are invisible. Another feature to test would be a scenario where the map and starting position is known but the robots cannot report their position. This would help compare the utility of the side panel force information against the added haptic force feedback.

\section{ACKNOWLEDGMENTS}

This research has been sponsored in part by AFOSR FA955008-10356 and ONR Grant N0001409-10680.

\section{REFERENCES}

Bashyal, S., \& Venayagamoorthy, G. (2008). Human Swarm Interaction for Radiation Source Search and Localization. . IEEE Swarm Intelligence Symposium, 1-8.

Coppin, G., \& Legras, F. (2012). Autonomy Spectrum and Performance Perception Issues in Swarm Supervisory Control. Proceedings of the IEEE, vol. 100, no. 3, 590-603.

Cummings M. (2004). Human Supervisory Control of Swarming Networks. 2nd Annual Swarming: Autonomous Intelligent Networked Systems Conference, 1-9.

Franchi, A., Giordano, P., Secchi, C., Son, H., \& Bulthoff, H. (2011). A Passivity-Based Decentralized Approach for the Bilateral Teleoperation of a Group of UAVs with Switching Topology. IEEE International Conference on Robotics and Automation, 898-905.

Gerkey, B., Vaughan, R., \& Howard, A. (2003). The Player/Stage Project: Tools for Multi-Robot and Distributed Sensor Systems. Proceedings of the 11th International Conference on Advanced Robotics, vol. 1. Portugal, 317-323.

Goodrich, M., Pendleton, B., Sujit, P., \& Pinto, J. (2011). Toward Human Interaction with Bio-Inspired Robot Teams. IEEE International Conference on Systems, Man, and Cybernetics, 2859-2864.

Howard, A., Mataric, M., \& Sukhatme, G. (2002). An Incremental SelfDeployment Algorithm for Mobile Sensor Networks. Autonomous Robots, vol. 13, no. 2, 113-126.

Kira, Z., \& Potter, M. (2009). Exerting Human Control Over Decentralized Robot Swarms. IEEE 4th International Conference on Autonomous Robots and Agents, 566-571.

Kolling, A., Nunnally, S., \& Lewis, M. (2012). Towards Human Control of Robot Swarms. Proceedings of the 7th International Conference on Human-Robot Interaction.

Lee, D., Franchi, A., Giordano, P., Son, H., \& Bulthoff, H. (2011). Haptic Teleoperation of Multiple Unmanned Aerial Vehicles Over the Internet. IEEE International Conference on Robotics and Automation, 13411347.

Naghsh, A., Gancet, J., Tanoto, A., and Roast, C. (2008). Analysis and Design of Human-Robot Swarm Interaction in Firefighting. Proceedings of 17th IEEE International Symposium on Robots and Human Interactive Communications, 255-260.

Nunnally, S., Walker, P., Kolling, A., Chakraborty, N., Lewis, M., Sycara, K., \& Goodrich, M. (2012). Human Influence of Robotic Swarms with Bandwidth and Localization Issues. International Conference on Systems, Man, and Cybernetics.

Quigley, M., Gerkey, B., Conley, K., Faust, J., Foote, T., Leibs, J., Berger, E., Wheeler, R., \& Ng, A. (2009). Ros: an Open-Source Robot Operating System. ICRA Workshop on Open Source Software, vol. 3, no. 3.2 .

Reynolds, Craig (1987), Flocks, herds and schools: A distributed behavioral model., SIGGRAPH '87: Proceedings of the 14th annual conference on Computer graphics and interactive techniques (Association for Computing Machinery): 25-34

Rodriguez-Seda, E., Troy, J., Erignac, C., Murray, P., Stipanovic, D., \& Spong, M. (2010). Bilateral Teleoperation of Multiple Mobile Agents: Coordinated Motion and Collision Avoidance. IEEE Transactions on Control Systems Technology, vol. 18, no. 4, 984-992.

Secchi, C., Franchi, A., Bulthoff, H., \& Giordano, P. (2012). Bilateral Teleoperation of a Group of UAVs with Communication Delays and Switching Topology. IEEE International Conference on Robotics and Automation, 4307-4314.

Walker, P., Kolling, A., Nunnally, S., Chakraborty, N., Lewis, M., Sycara, K., \& Goodrich, M. (2012). Neglect Benevolence in Human Control of Swarms in the Presence of Latency. International Conference on Systems, Man, and Cybernetics. 